\title{
Neighbor Selection Techniques for Multi-Hop Wireless Mesh Networks
}

\author{
Baldomero Coll and Javier Gozalvez \\ Uwicore, Ubiquitous Wireless Communications Research Laboratory, http://www.uwicore.umh.es \\ University Miguel Hernandez of Elche \\ Avda. de la Universidad, s/n, 03202 Elche, Spain \\ bcoll@umh.es,j.gozalvez@umh.es
}

\begin{abstract}
Multi-hop wireless mesh networks, including MultiHop Cellular Networks, base their operation on the establishment of multi-hop paths to route the information from source to destination. To this aim, it is crucial to define adequate neighbor selection techniques that choose the most appropriate nodes over which to route the information. In this context, this paper proposes a set of neighbor selection techniques that exploit spatial diversity to improve the performance and operation of multi-hop wireless mesh networks.
\end{abstract}

Keywords-component: neighbor selection, multi-hop communications, mesh networking, 802.11s, multi-hop cellular networks.

\section{INTRODUCTION}

Future 4G cellular systems are required to provide high and homogeneous bit rates over the complete cell coverage area. Traditional cellular architectures, where each Mobile Station (MS) directly communicates with the Base Station (BS), are not capable to provide such homogeneous high bit rates due to the signal attenuation with the increasing distance. Achieving such homogenous and high bit rates requires installing a higher number of base stations, or integrating cellular and ad-hoc networking technologies. The integration of cellular and ad-hoc technologies, also referred as Multi-hop Cellular Network (MCN) [1], has gained significant research attention given its capability to achieve the $4 \mathrm{G}$ objectives by substituting a direct MS-BS link by multiple hops using either fixed relays (MCNFixed Relay, MCN-FR) or mobile relays (MCN-Mobile Relay, MCN-MR). Using multiple hops reduces the communications distance and signal loss in each hop, thereby offering the possibility to increase the overall multi-hop transmission rate.

IEEE 802.11s standard [2] is a suitable candidate for the multi-hop ad-hoc operation of MCN-MR networks since it incorporates all networking functions necessary for the establishment of wireless mesh networks. A key aspect in the operation of multi-hop wireless mesh networks is the identification and selection of neighboring mesh nodes (MP, Mesh Point) that will be in charge of routing the information from source to destination. Such routing nodes are referred in the 802.11s standard as peer MPs or neighbor peer MPs. Various ad-hoc routing studies (e.g. [3]) have demonstrated the importance and impact of the neighbor's selection on the operation and performance of multi-hop routing protocols. For example, an adequate neighbors selection technique can help reducing the signalling load necessary to establish multi-hop mesh routes. In this context, it is important to highlight that the 802.11 s standard clearly indicates that the number of neighbor peer MPs must be limited, but does not define the techniques to be implemented for their selection.

Initial proposals to select the neighbor peer MPs used selection criteria based on the received signal level from neighboring nodes [4], or the node's proximity [5]. These proposals did not consider the particular characteristics of urban environments where non uniform node's density distribution and frequent non line of sight propagation conditions might be experienced. In this environment, an adequate selection of neighbor peer MPs is even more crucial to increase the nodes connectivity and ensure that a multi-hop routing path from source to destination can be established. A recent proposal considering the specific characteristics of urban environments has been proposed in [6], where the authors introduce the Friend Management Algorithm (FMA) designed for each node to select stable neighbors in urban ad hoc networks using their beaconing messages. In particular, the authors base their neighbor selection process on Packet Error Rate (PER) estimates of beacon messages. Using such estimates and the urban mobility knowledge, the FMA proposal filters unreliable neighbors considering the characteristics of urban mobility; for example, nodes moving in the opposite direction. The authors claim that their proposal ensures that the nodes that would be neighbors for a longer period of time considering urban mobility patterns are selected as neighbor nodes.

Despite these interesting recent advancements, it is important to note that all these proposals did not considered the implementation constraint that only a limited number of neighbor peer MPs can be selected. In addition, the proposals only focus on the local neighbor selection process, but do not look at whether such local neighbor selection decision would allow guaranteeing that a reliable multi-hop route from source to destination can be established. In this context, this work proposes and evaluates a set of new neighbor selection techniques that exploit spatial diversity to increase the node's forward connectivity. In addition, the proposed technique implement the 802.11 s constraint that only a limited set of neighbor nodes can be selected. As it will be demonstrated, the 
proposed spatial diversity approach is capable to guarantee the establishment of diverse multi-hop paths that increase the probability to reliably route the information from source to destination.

The rest of this paper is organized as follows. In Section II a fundamental explanation about the IEEE 802.11s standard is provided. Our proposed neighbor selection techniques are introduced in Section III. The simulation environment and corresponding performance evaluation of the neighbor selection techniques are shown in Section IV and $\mathrm{V}$, respectively. Finally, the conclusion of the research work is given in Section VI.

\section{IEEE 802.11s STANDARD}

This work is based on the IEEE 802.11s standard that introduces new networking functions for the establishment of wireless mesh networks, including the mesh discovery process, authentication, mesh links management, channel selection, security, mesh route establishment and congestion control among others. In this work, we focus on the mesh discovery and management processes.

The mesh network discovery process is enabled through the periodic broadcast exchange of beaconing messages among neighboring nodes. The routing protocol proposed in the 802.11s standard is the Hybrid Wireless Mesh Protocol (HWMP), that includes both a reactive and proactive operational mode. In this paper, we focus on the reactive modified version of the AODV (Ad-Hoc On-Demand Distance Vector) [7] protocol that is part of HWMP. AODV is a reactive routing protocol that only searches and establishes a route from source to destination when the source has information to transmit and does not know the route to reach the destination node. In this case, the source node sends a broadcast Route REQuest (RREQ) message that is retransmitted by neighboring nodes. When the destination node receives the RREQ message, it replies with a unicast Route REPly (RREP) message to confirm the route establishment. The reception of RREQ and RREP messages allow intermediate nodes to know their neighboring nodes in the route towards the source and destination nodes.

In the original AODV protocol, the route selected between the source and destination nodes is that with the lower latency, which generally coincides with the route with the lowest number of hops from source to destination. To consider other network parameters, this work is based on the modified AODV protocol, and in particular the on-demand routing mechanism included in the 802.11s standard [2]. The modified AODV implementation still allows the lowest latency route to be created. However, when another RREQ with the same sequence number is received by a node, instead of being discarded, the modified AODV protocol evaluates whether the new route's cost is lower or higher than the route that has already been identified, with the cost computed following a cost function previously defined. To compute the cost of each peer link, the 802.11 s standard defines the Airtime Link metric but allows for other metrics and cost functions to be implemented. This work is based on the Multiple Metrics (MM) cost function proposed in [8], which bases its routing decision on the number of hops, the channel congestion and the energy consumption. This cost function has been adopted in this work since it considers some of the parameters that are more important for the performance and operation of multi-hop wireless mesh networks, including MCN-MR networks.

The mesh peer link management mechanism described in the $802.11 \mathrm{~s}$ standard handles the establishment and breakage of peer links betweens MPs. MPs shall not transmit data frames or management frames other than the ones used for the mesh discovery and peer link management until a peer link with a neighboring node has been established. Once such peer link is successfully established between two nodes, they mutually call each other neighbor peer MPs or peer MPs. Although the 802.11 s standard indicates that the number of neighbor peer MPs must be limited, it does not define the process to identify and select candidate peer MPs. To ensure that no node remains isolated from a mesh network, the 802.11s standard indicates that every MP must be capable to establish at least one peer link with other MPs within its radio range, even if they do not comply with the peer MP selection criteria.

Figure 1 illustrates the 802.11s message exchange to establish and break peer links between MPs. MP1 initiates a request to establish a peer link with node $M P 2$ by sending a $P L$ request (PeerLink request) message. $M P 2$ replies to this request with the $P L \_$confirm confirmation message announcing its willingness to establish a peer link with node $M P 1$. This message exchange is then repeated by $M P 2$, and a peer link is successfully established between two nodes when they both have sent, received and correctly processed the $P L$ request and $P L$ confirm messages. The process to break a peer link established between peer MPs is initiated with the transmission of a PL close message. The receipt of such message breaks an established peer link, and the receiving node sends a $P L \_$close_confirm message to indicate its closure acceptance and complete the peer link breakage process. Figure 1 also represents the possible states of the MPs that participate in the establishment and breakage of peer MP links:

- $\quad$ LISTEN: MPs are listening for the potential receipt of a $P L$ request message to establish a peer link.

- OPN_SNT: an MP has sent a PL_request message and is waiting for the receipt of the $P \bar{L}_{-}$confirm reply.

- CNF_RCVD: an MP has received the PL_confirm reply but has not yet received the $P L \_$request from the MP with which it is looking to establish a peer link.

- OPN RCVD: this state is reached by the MP with which we are looking to establish a peer link, when it has received a $P L$ request message but it has not yet received the $P L_{-}$confirm reply to its $P L_{-}$request message.

- ESTAB: this state is reached when an MP completes the peer link establishment process with another node (the MP has received and sent the PL_request and $P L \_$confirm messages).

- HOLDING: an MP is in the process to break a peer link with another node, and it is waiting to receive the PL_close_confirm message. 


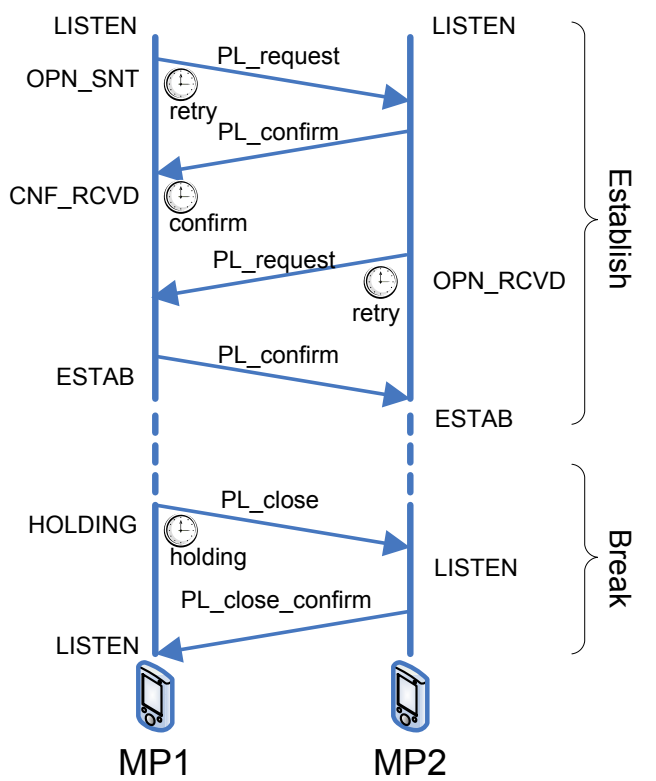

Figure 1. Messages exchanged in a peer link management process.

The peer link establishment process defines timers to avoid permanently waiting for reply messages. The timers participating in the peer link management process are also illustrated in Figure 1. The retry timer controls the maximum waiting time for a PL_confirm message after a PL_request. The confirm timer defines the maximum waiting time to receive a PL_request after a PL_confirm message. If any of these timers expire, the peer link establishment process between two MPs is ended, and they both return to their LISTEN state. Finally, the holding timer is initiated at the start of the process to break a peer link, and avoids an excessive waiting time for a PL_close_confirm message. If such message is not received by the time the holding timer expires, the peer link is broken.

\section{NEIGHBOR SELECTION TECHNIQUES}

The proposed neighbor selection techniques follow a two phase operation. During the establishment phase, each MP executes with a $T$ periodicity the mesh peer link establishment mechanism with its neighbors ${ }^{1}$. The establishment phase is run only if a node has fewer neighbor peer MPs than that allowed (max_peer_MPs). The update phase checks that the selected neighbor peer MPs still fulfil the neighbor peer MP selection criteria. If this is not the case, and there is a another node that fulfils such criteria, this node will then replace the neighbor peer MP that does not fulfil anymore the neighbor selection criteria. The update phase is run in this work with a $3 T$ periodicity.

\section{A. Bidirectional Neighbor Selection (BiNS) technique}

The Bidirectional Neighbor Selection (BiNS) technique is aimed at exploiting spatial diversity to provide varied routes between the source and destination nodes. To do so, BiNS avoids selecting all neighbor peer MPs in the same direction towards the destination node. During the establishment phase, BiNS selects all its neighbor peer MPs, except the last one, following the beacon's message PER estimate proposed in [6], and that will be hereafter referred to as PER algorithm. In our implementation, the PER algorithm selects the neighbors with a lower PER estimate, measured over the last 20 beacon periods. To select the last neighbor peer MP, BiNS checks the location of the already selected neighbor peer MPs, and selects a last neighbor peer MP that is located in the opposite direction of the previously selected neighbor peer MPs. The proposed technique requires that all neighbors transmit their position in the beacon messages, which is not unrealistic given the continuous increase of devices with embedded GPS capabilities. The update phase is only performed if the BiNS criteria (i.e. all the selected neighbor peer MPs are not in the same direction) is not fulfilled and there is a candidate peer MP that could fulfill it.

The BiNS operation is illustrated in Figure 2. Let's suppose that the maximum number of neighbor peer MPs is four. The first three neighbor peer MPs $(M P 1, M P 2$ and $M P 3$ in the figure) are selected following the PER criteria; for the example illustrated in Figure 2, we consider that the neighbor peer MPs with the best PER performance are those at a shortest distance from the source node $S$. If we applied the PER criteria to select the fourth and last neighbor peer MP, MP4 would be the last selected neighbor peer MP. However, selecting MP4 as the last neighbor peer MP would locate west of the source node $S$ all the selected neighbor peer MPs, which would limit the possibilities to reach the destination node, if such node was located east of the source node $S$. In this context, the BiNS technique selects MP5 as the last neighbor peer MP.

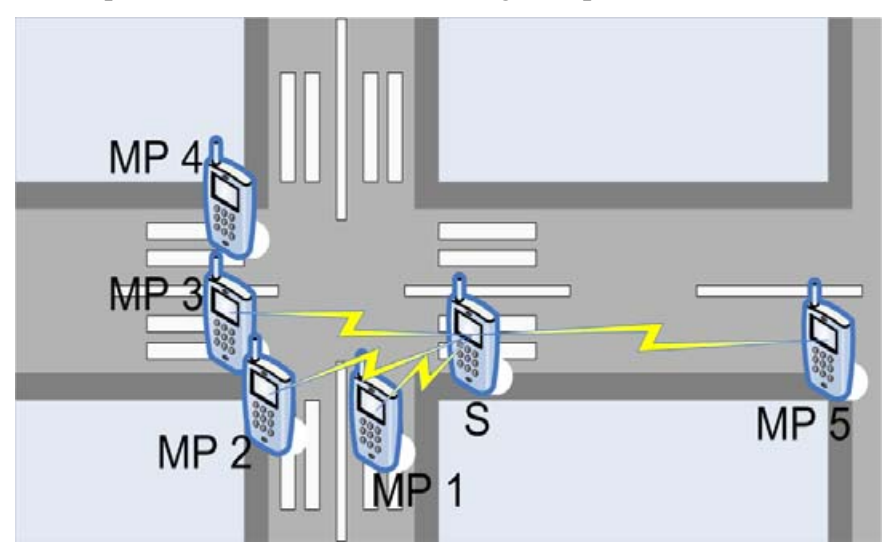

Figure 2. BiNS neighbor peer MP selection process.

\section{B. Minimum Separation Neighbor Selection (MiSeNS) technique}

The Minimum Separation Neighbor Selection (MiSeNS) proposal also targets to exploit spatial diversity in the neighbor peer MP selection process by avoiding selecting closely located neighbor peer MPs. To this aim, the MiSeNS proposal defines a minimum separation (dist_PEERs) that must be guaranteed between the node that is selecting neighbors and all its neighbor MPs, and also between the selected neighbor peer

\footnotetext{
${ }^{1} T$ represents the beacon's exchange periodicity.
} 
$\mathrm{MPs}^{2}$. This technique is proposed since selecting closely located neighbor peer MPs would not provide the multi-hop route diversity that is expected to improve the operation and performance of multi-hop wireless mesh networks.

During the establishment phase, MiSeNS selects all its neighbor peer MPs following the PER algorithm as long as the minimum separation criteria previously described is guaranteed. The protocol would then verify during the update phase if the minimum distance criteria is still guaranteed. If not, the MiSeNS technique would replace the neighbor peer MPs that do not guarantee the minimum separation criteria. The MiSeNS operation is illustrated in Figure 3, where dist_PEERs has been set equal to the street's width. If the PER algorithm was applied ${ }^{3}, M P 1, M P 2, M P 3$ and $M P 4$ would have been selected as neighbor peer MPs. Since $M P 2$ and $M P 3$ are closely located to $M P 1$, and hence, do not provide any multihop route diversity, MiSeNS discards them as potential neighbor peer MPs and selects instead the nodes MP5 and MP6 as neighbor peer MPs.

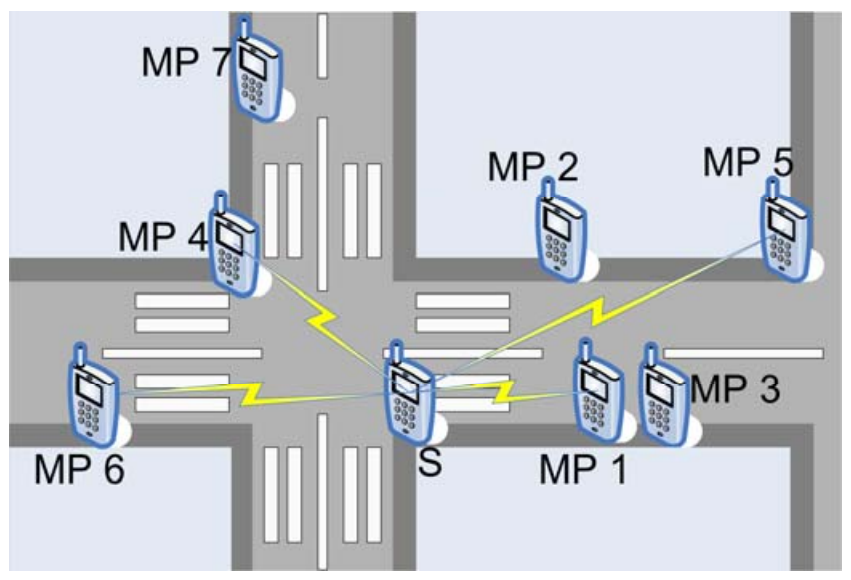

Figure 3. MiSeNS neighbor peer MP selection process.

\section{Bidirectional and Minimum Separation Neighbor Selection (BiMiSeNS) technique}

Finally, this paper proposes a third spatial diversity neighbor selection technique that combines the BiNS and MiSeNS proposals to increase the wireless multi-hop mesh route diversity. During the establishment phase, the Bidirectional and Minimum Separation Neighbor Selection (BiMiSeNS) technique selects all the neighbor peer MPs following the PER algorithm and the minimum separation criteria. If all the selected neighbor peer MPs are located in the same direction, the BiMiSeNS proposal selects the last neighbor peer MP so that it is located in the opposite direction of the other neighbor peer MPs, while still guaranteeing the minimum separation criteria. In the update phase, BiMiSeNS checks first that the bidirectional criteria is guaranteed with the

\footnotetext{
2 As a result, the MiSeNS proposal also requires transmitting the node's position in the beacon message.

${ }^{3}$ For the example illustrated in Figure 3, we also assume that the maximum number of neighbor peer MPs is four, and that the neighbor peer MPs with the best PER performance are those at a shortest distance from the source node $S$.
}

selected neighbor peer MPs, and then the minimum separation criteria.

\section{SimUlation ENVIRONMENT}

This work has been conducted using the ns 2 simulation platform. The version employed is ns 2.29 that incorporates the mobility extension developed by CMU's Monarch Group [9], which allows the simulation of WLAN networks as well as adhoc multi-hop networks. The main CMU's Monrach Group contributions to the mobility extension are: the implementation of the Address Resolution Protocol (ARP), the implementation of some ad hoc network routing protocols such as DSR, DSDV, TORA and AODV; and the support for introducing new MAC layer implementations.

In particular, a $1900 \mathrm{~m} \times 1900 \mathrm{~m}$ Manhattan scenario with 350 nodes moving following the Random Walk Obstacle [10] mobility model has been emulated. In Random Walk Obstacle model each node moves along the street graph. At each corner, the node selects the neighboring corner at random and moves towards it. In this work, the maximum number of neighbor peer MPs (max_peer_MPs) that can be selected has been set equal to four. In this case, the emulated mobile node's density guarantees that $95 \%$ of the nodes have at least four neighbors to select from, which ensures that the neighbor peer MPs can be selected following the neighbor selection techniques under study.

The nodes communicate using the IEEE802.11a standard at $5.8 \mathrm{GHz}$. The propagation loss is modelled through the deterministic pathloss model implemented in the WINNER European project for the urban micro-cellular scenario. This model differentiates the path loss experienced under Line of Sight (LOS) and Non LOS (NLOS) conditions [11], which are highly influenced in this work by the presence of obstructing buildings. Despite not considering equal transmitting and receiving antenna heights, to the author's knowledge this model is the most suitable one since it reproduces urban environment with relatively low BS antenna heights.

The WINNER pathloss for LOS conditions is expressed as follows:

$$
P L_{\text {Los }}(d[m])=\left\{\begin{array}{c}
22.7 \log _{10}(d[m])+41+20 \log _{10}(f[G H z] / 5) \\
\text { if } \quad d<R_{b p} \\
40 \log _{10}(d[m])+41-17.3 \log _{10}\left(R_{b p}\right)+20 \log _{10}(f[G H z] / 5) \\
\text { if } \quad d \geq R_{b p}
\end{array}\right.
$$

where:

$$
R_{b p}=4 \frac{\left(h_{A}-1\right)\left(h_{B}-1\right)}{\lambda}
$$

$d$ is de distance between the transmitter and the receiver, $h_{A}$ and $h_{B}$ are their respective antenna heights and $f$ is the carrier frequency.

For NLOS conditions, the pathloss can be expressed as: 


$$
\begin{aligned}
P L_{\text {NLOS }}\left(d_{A}[m], d_{B}[m]\right)= & P L_{L O S}\left(d_{A}[m]\right)+20-12.5 n_{j}+ \\
& +10 n_{j} \log _{10}\left(d_{B}[m]\right)
\end{aligned}
$$

where:

$$
n_{j}=\max \left(2.8-0.0024 d_{A}[m], 1.84\right)
$$

$d_{A}$ and $d_{B}$ are the transmitter and the receiver distances to the closest intersection.

Figure 4 shows the related LOS-NLOS condition in urban scenario.

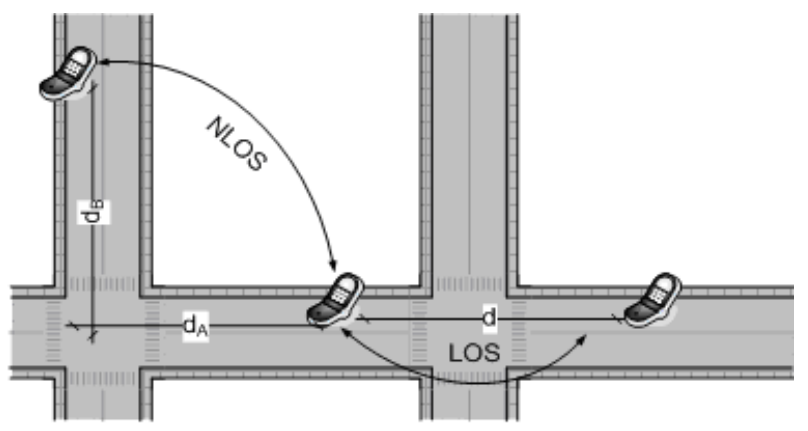

Figure 4. Propagation conditions in a realistic urban environment.

The implemented traffic model follows the on-off bursty pattern characteristic of data transmissions, but does not try to exactly characterise any specific traffic source. In particular, the model simulates 200 seconds traffic sessions where on and off periods last 5 and 15 seconds respectively. The defined off period ensures that the nodes routing tables validity has expired at the start of the following on period. During the on period, the source nodes transmit 50 packets to the destination node that is located at the centre of the simulated Manhattan scenario. Figure 5 summarizes the on-off data traffic model.

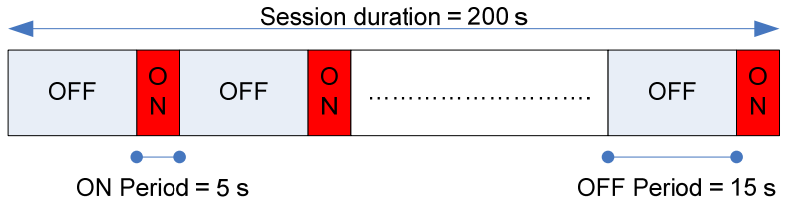

Figure 5. On-Off data traffic model.

The remaining simulation platform parameters are summarised in Table I.

TABLE I. NS2 SIMULATION PARAMETERS

\begin{tabular}{|l|c|}
\hline \multicolumn{1}{|c|}{ Parameter } & Value \\
\hline Number of nodes & 350 \\
\hline Buildings width (m) & 225 \\
\hline Street width (m) & 25 \\
\hline dist_PEERs (m) & 25 \\
\hline Transmission power (W) & 0.2 \\
\hline Transmission rate (Mbps) & 12 \\
\hline Node's speed (m/s) & 1.5 \\
\hline Data packet size (bytes) & 500 \\
\hline Beacon's period (s) & 1 \\
\hline Simulation time (s) & 10000 \\
\hline
\end{tabular}

\section{PERFORMANCE EVALUATION}

The performance of the proposed neighbor selection techniques is compared to that achieved using the PER algorithm, and considering an unlimited number of neighbor peer MPs, hereafter referred as PER and MM respectively. At this stage, it is important to note that although considering all neighbors as potential neighbor peer MPs is not realistic, this case has been simulated to achieve the upper bound benchmark reference performance over which to compare the neighbor selection techniques proposed in this paper.

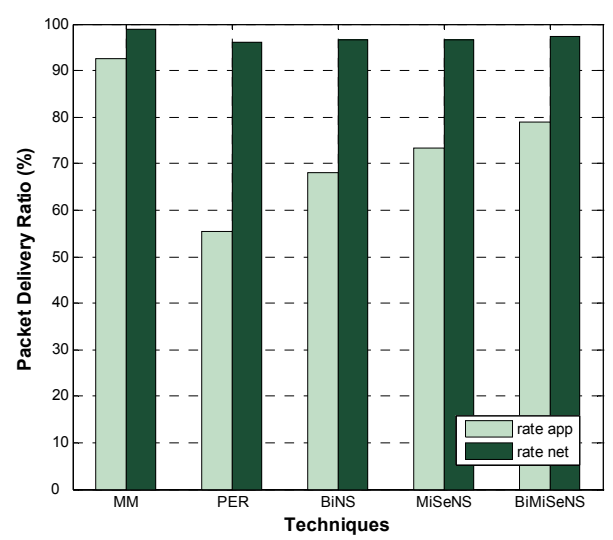

Figure 6. Packet Delivery Ratio at the application and network levels.

TABLE II. NUMBER OF RETRANSMITTED RREQ MESSAGES PER ROUTE ESTABLISHMENT

\begin{tabular}{|l|c|}
\hline \multicolumn{1}{|c|}{ Techniques } & RREQ/route \\
\hline MM & 472.33 \\
\hline PER & 54.37 \\
\hline BiNS & 78.43 \\
\hline MiSeNS & 86.03 \\
\hline BiMiSeNS & 101.59 \\
\hline
\end{tabular}

Figure 6 shows that an unlimited number of neighbor peer MPs (MM technique) would achieve the highest packet delivery ratios, both at the application level (rate app) and the network level (rate_net) ${ }^{4}$. However, considering all nodes as potential neighbor peer MPs significantly increases the signalling load associated with the multi-hop route establishment. In fact, the results depicted in Table II show that MM considerably increases (465\% and $600 \%$ with respect to BiMiSeNS and BiNS respectively) the number of retransmitted RREQ packets per route establishment. The results illustrated in Figure 6 also show that the neighbor selection criteria exploiting spatial diversity increase the application packet delivery ratio, with the increase augmenting with the spatial diversity. In fact, BiMiSeNS is the technique achieving the best performance, improving the application packet delivery ratio by $45 \%$ compared to the PER technique. As shown in Table III, the techniques achieving the highest performance are those resulting in a higher spatial diversity. The results depicted in

\footnotetext{
${ }^{4}$ The rate_net parameter represents the packet delivery ratio to the destination node with regards to the routed packets.
} 
Figure 6 and Table III show that the neighbor selection techniques increasing the spatial diversity improve the probability to find a multi-hop route from source to destination. In addition, the proposed techniques also increase the duration and stability of the peer mesh links compared to the PER technique (Figure 7), although in this case the duration slightly decreases as the spatial diversity increases.

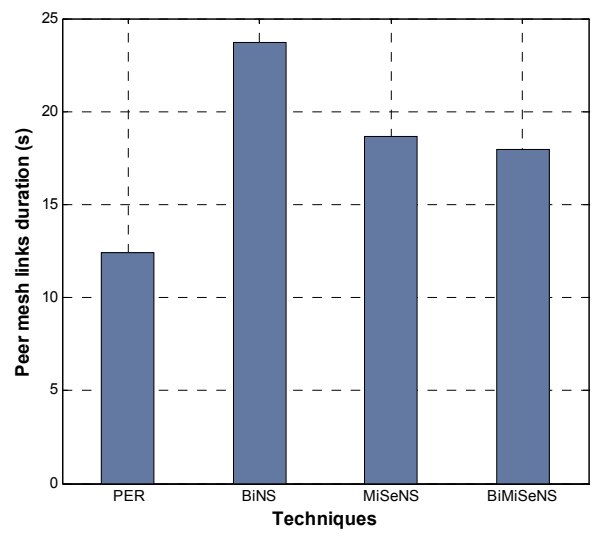

Figure 7. Neighbor peer MPs link's duration.

TABLE III. MEAN AND STANDARD DEVIATION OF THE DISTANCE BETWEEN SELECTED NEIGHBOR PEER MPS.

\begin{tabular}{|l|c|c|c|c|}
\hline Technique & PER & BiNS & MiSeNS & BiMiSeNS \\
\hline Mean & 150.62 & 159.76 & 182.01 & 183.42 \\
\hline Standard deviation & 110.12 & 113.62 & 118.24 & 118.32 \\
\hline
\end{tabular}

Table IV highlights some of the main characteristics of the multi-hop routes established using the implemented neighbor selection techniques. The illustrated results clearly show that limiting the number of possible neighbor peer MPs is not only necessary for a realistic multi-hop wireless mesh implementation, but also significantly conditions the operation of such networks since it increases the number of hops and distance from source to destination. In the results illustrated in Table IV, MM increases the distance between hops since the original MM cost function favours the establishment of routes with the lower possible number of hops. Table IV also shows that when the PER algorithm manages to establish multi-hop routes from source to destination, these routes have a shorter length than the proposed neighbor selection techniques. However, the PER technique increases the time ${ }^{5}$ and signalling needed to establish such multi-hop routes, and reduces their duration. The difficulty of the PER technique in establishing a route from source to destination compared to the proposed neighbor selection techniques is also observed in the increase in the number of RREQ messages necessary to establish a multi-hop route. The AODV routing protocol retransmits a

\footnotetext{
5 The time needed to establish a multi-hop route is measured as the time difference between the first RREQ message sent by the source node and the reception of the RREP message by the source node.
}

RREQ message if a RREP message is not received before a timer expires. Increasing the number of RREQ messages transmitted per created route indicates a higher difficulty to establish the route from source to destination. The proposed neighbor selection techniques also increase the multi-hop routes duration compared to the PER technique, and even to the case in which an unlimited number of neighbor peer MPs can be selected. Another interesting parameter reflecting the achieved multi-hop route diversity with the techniques proposed in this paper is the ratio between the number of received RREP messages and the number of transmitted RREQ messages. In fact, the results obtained show that the proposed techniques seeking to have neighbor peer MPs in opposite directions (BiNS and BiMiSeNS) result in a higher number of RREP messages per route requested, which highlights the achieved spatial diversity.

TABLE IV. MULTI-HOP ROUTE CHARACTERISTICS

\begin{tabular}{|l|c|c|c|c|c|}
\hline \multicolumn{1}{|c|}{ Technique } & MM & PER & BiNS & MiSeNS & BiMiSeNS \\
\hline Number of hops & 6.55 & 14.4 & 14.7 & 14.0 & 13.9 \\
\hline Hop distance (m) & 185.7 & 122.2 & 122.2 & 140.0 & 139.1 \\
\hline Multi-hop route distance (m) & 1157.3 & 1746.1 & 1771.3 & 1936.0 & 1908.6 \\
\hline $\begin{array}{l}\text { Time to establish a multi- } \\
\text { hop route (s) }\end{array}$ & 0.041 & 0.230 & 0.199 & 0.182 & 0.209 \\
\hline RREQ needed per route & 1.12 & 1.98 & 1.73 & 1.58 & 1.77 \\
\hline Route duration (s) & 1.55 & 1.47 & 1.61 & 1.70 & 1.53 \\
\hline Percentage of broken routes & 25.55 & 31.41 & 30.35 & 35.99 & 32.65 \\
\hline RREP / RREQ & 1.64 & 1.28 & 1.42 & 1.29 & 1.37 \\
\hline
\end{tabular}

TABLE V. ENERGY CONSUMPTION PER NODE

\begin{tabular}{|l|c|c|c|c|}
\hline Technique & $\begin{array}{c}\text { E_data } \\
(\mathbf{J})\end{array}$ & $\begin{array}{c}\text { E_routing } \\
(\mathbf{J})\end{array}$ & $\begin{array}{c}\text { E_link } \\
(\mathbf{J})\end{array}$ & $\begin{array}{c}\text { E_beacon } \\
(\mathbf{J})\end{array}$ \\
\hline PER & 1.02 & 0.66 & 1.02 & 0.51 \\
\hline BiNS & 1.28 & 0.73 & 0.65 & 0.51 \\
\hline MiSeNS & 1.33 & 0.70 & 0.92 & 0.51 \\
\hline BiMiSeNS & 1.42 & 0.69 & 0.94 & 0.51 \\
\hline
\end{tabular}

Finally, the results illustrated in Table $\mathrm{V}$ highlight the energy consumption per node comparison for the implemented neighbor selection techniques. The E_beacon parameter represents the energy consumed by the beaconing messages used to discover and establish the mesh network. The E_beacon result is equal to all the simulated techniques since they all implemented the same beacon periodicity. The E_link parameter reflects the energy consumption in the establishment and destruction of peer mesh links. The shorter peer MP link duration measured with the PER technique (Figure 5) results in a higher peer mesh link management energy consumption. The E_routing parameter measures the energy consumption derived from all tasks needed to establish a multi-hop route (e.g. the transmission of RREQ and RREP messages). The achieved E_routing value for all the simulated techniques is very similar given that the off period in the simulated traffic model always 
requires defining a new multi-hop route from source to destination for every on data period. Finally, E_data represents the energy consumption derived from the transmission and reception of data packets. As it could be expected, the neighbor selection techniques increasing the application packet delivery ratio result in higher data energy consumption.

\section{CONCLUSIONS}

This work has proposed and evaluated a set of new neighbor selection techniques exploiting spatial diversity and designed to be easily implemented in mesh networking standards such as $802.11 \mathrm{~s}$. The achieved results have demonstrated that exploiting such spatial diversity improves the performance and operation of multi-hop wireless mesh networks, including their stability, route duration and energy consumption.

\section{ACKNOWLEDGMENTS}

This work has been supported by the Spanish Ministry of Science and Innovation, the Spanish Ministry of Industry, Tourism and Commerce and FEDER funds under the projects TEC2008-06728 and TSI-02400-2008-113.

\section{REFERENCES}

[1] Y. Lin and Y. Hsu, "Multihop Cellular: A New Architecture for Wireless Communications", in IEEE Proceedings Computer Communications Conference (INFOCOM), vol. 3, pp. 1273-1282, 2000, Israel.
[2] IEEE P802.11s/D2.0, draft amendment to standard IEEE 802.11: Mesh Networking. IEEE Standard, 2007.

[3] R. Sisodia, B. Manoj and C. Murthy, "A preferred link based routing protocol for ad hoc wireless networks", Journal of Communications and Networks, Vol. 4, No. 1, pp. 14-21, 2002.

[4] S. Itaya, J. Hasegawa, P. Davis, N. Kadowaki and S. Obana, "Achieving stable operation of ad hoc wireless networks with neighbor pre-selection and synchronous route updates", in IEEE Proceedings Local Computer Networks Conference (LCN), pp. 702-708, 2005, Australia.

[5] C. Cramer and T. Fuhrmann, "Proximity Neighbor Selection for a DHT in Wireless Multi-Hop Networks", in IEEE Proceedings Peer-to-Peer Computing Conference (P2P), pp. 3-10, 2005, Germany.

[6] A. Uchiyama, S. Fujii, T. Umeda, H. Yamaguchi and T. Higashino, "Neighbor Selection Algorithm for Ad hoc Networks with Highly Dynamic Urban Mobility", in Proceedings International Wireless Communications and Mobile Computing Conference (IWCMC), pp. 165170, 2008, Greece.

[7] C. Perkins and E. Royer, "Ad hoc On-Demand Distance Vector Routing", in IEEE Proceedings Workshop on Mobile Computing Systems \& Applications (WMCSA), pp. 90-100, 1999, USA.

[8] L. Cao, K. Sharif, Y. Wang and T. Dahlberg, "Adaptive Multiple Metrics Routing Protocols for Heterogeneous Multi-Hop Wireless Network", in IEEE Proceedings Consumer Communications \& Networking Conference (CCNC), pp. 13-17, 2008, USA.

[9] CMU , $\mathrm{s}$ Monarch group. Sitio web official: http://www.monarch.cs.rice.edu/

[10] K. Maeda, A. Uchiyama, T. Umedu, H. Yamaguchi, K. Yasumoto and T. Higashino, "Urban Pedestrian Mobility for Mobile Wireless Network Simulation", in Ad Hoc Networks, Elsevier, Vol. 7, No. 1, pp. 153-170, 2009.

[11] WINNER, "D1.1.1. WINNER II interim channel models", Public Deliverable, https://www.ist-winner.org/index.html 